\title{
Effect of filtering in dense WDM metro networks adopting VCSEL-based multi-Tb/s transmitters
}

\author{
P. Parolari ${ }^{1}$ Member, IEEE, A. Gatto ${ }^{1}$, M. Rapisarda ${ }^{1}$, C. Neumeyr ${ }^{2}$, M. Svaluto Moreolo ${ }^{3}$ Senior Member, \\ IEEE, J.M. Fabrega ${ }^{3}$ Senior Member, IEEE, L. Nadal ${ }^{3}$ Senior Member, IEEE, P. Boffi ${ }^{1}$ Member, IEEE \\ ${ }^{1}$ Politecnico di Milano, Dip. Elettronica Informazione e Bioingegneria, Via Ponzio 34/5,20133 Milano, Italy \\ ${ }^{2}$ VERTILAS GmbH, Daimlerstr 11d, D-85748 Garching, Germany \\ ${ }^{3}$ Centre Tecnolòic de Telecomunicacions de Catalunya (CTTC/CERCA), Av. C. F. Gauss 7, 08860 Castelldefels \\ (Barcelona), Spain \\ e-mail: paola.parolari@polimi.it
}

\begin{abstract}
Long-wavelength vertical cavity surface emitting lasers (VCSELs) can represent an alternative solution for the development of transmitters with reduced cost, power consumption and footprint for very-high capacity metropolitan area systems. Multi-Tb/s transmitter modules with fine wavelength division multiplexing (WDM) granularity can be obtained adopting direct modulation (DM) with advanced modulation formats, such as discrete multitone (DMT), and aggregating multiple DM-VCSELs emitting in the C-band with WDM multiplexers in SOI chips. Due to numerous hops between nodes inside metropolitan area networks the effect of filtering can severely impact the transmission performance; we evaluate the transported capacity in function of nodes number taking into account the actual VCSEL parameters and simplified coherent detection.
\end{abstract}

Keywords: VCSELs, metro networks, WSS, discrete multitone transmission.

\section{INTRODUCTION}

Metropolitan area networks (MANs) are evolving toward more efficient and agile solutions able to support the IP traffic increase and its uneven distribution along day-time. Services such as 5G, Mobile Edge Computing, UHD $\mathrm{TV}$, need a MAN allowing multi- $\mathrm{Tb} / \mathrm{s}$ transmission and routing over variable distances and topologies, supporting a "pay-as-you-grow" evolution, to target reduced costs and possibly power consumption [1].

The development of tailored MAN photonic technologies could overcome present limits of traditional highcapacity systems targeting hundreds of $\mathrm{Gb} / \mathrm{s}$ capacity per link, which have been designed for long-haul transport networks and have not completely addressed cost, consumption and scalability issues.

A sliceable bandwidth/bitrate variable transceiver (S-BVT) based on vertical cavity surface emitting laser (VCSEL) can represent an innovative approach for MANs [2]. Long-wavelength InP VCSELs emitting in the C band directly modulated (DM) by multicarrier formats, such as discrete multitone (DMT), have been recently proposed as a cost-effective and energy-efficient solution to reach high capacities over tens of kms [3-5].

The requested MAN traffic scaling can be otherwise obtained by exploiting a modular approach in the realization of the VCSEL-based S-BVT transmitters. Tens of DMT-DM-VCSELs can be integrated in a single Silicon-oninsulator (SOI) chip with dense wavelength division multiplexers to provide $25-\mathrm{GHz}$ fine granularity and handle multi- $\mathrm{Tb} / \mathrm{s}$ capacities $[6,7]$. Moreover, in order to target typical MAN reach distances in standard single mode fiber (SSMF) links, the cumulated chromatic dispersion (CD) can be compensated by exploiting digital coherent detection (COHD) [8].

The single high-bandwidth VCSEL can be directly modulated to achieve a rate higher than $50 \mathrm{~Gb} / \mathrm{s}$ per wavelength, obtained by single side-band (SSB) DMT modulation, achieving, in case of polarization division multiplexing (PDM), a spectral efficiency of $4 \mathrm{~b} / \mathrm{s} / \mathrm{Hz}$. Due to chirp associated with DM and to the adopted fine wavelength division multiplexing (WDM) granularity, filtering effects need to be considered [9].

In this paper we assess the performance of the above-mentioned transmitter, employing coherent detection with reduced complexity. In particular we take into account the impact of traversing multiple nodes, based on wavelength selective switching (WSS) filters inside metropolitan area networks. We evaluate the transported capacity for multichannel transmission in function of nodes number, considering the actual VCSEL chirp coefficients and frequency response [9] and we demonstrate reach distances of hundreds of kms typical of MANs, maintaining a capacity per channel higher than $50 \mathrm{~Gb} / \mathrm{s}$.

\section{VCSEL-BASED S-BVT TRANSMITTER AND SIMPLIFIED COHERENT RECEIVER}

The analyzed transmitter is based on a modular approach: each module is constituted by a SOI chip integrating multiple VCSELs at operating wavelengths within the C-band with 100-GHz channel spacing $[1,6]$. The fine granularity of $25 \mathrm{GHz}$ is achieved by interleaving 4 modules and the efficient exploitation of the spectral resource is performed by DM high-bandwidth short-cavity (SC) VCSELs, with SSB DMT modulation. Thanks to bit and power loading at the digital signal processing (DSP) level [10], DMT is capable of addressing the efficient usage 
of the bandwidth resource as a function of the requested capacity and transmission distance, allowing dynamic and flexible adaptation to traffic/channel conditions and spectrum fragmentation mitigation in MANs [11].

The SC VCSELs are characterized by an E/O bandwidth of $20 \mathrm{GHz}$; as the most significant drawback of the direct modulation of VCSELs is the frequency chirp, reliable chirp parameters were obtained by preliminary measurements providing a $\alpha$ linewidth enhancement factor of 3.7 and $\kappa$ adiabatic constant of $1.52 \cdot 10^{13}$ [9].

The DMT signal is composed by 256 sub-carriers, different frequency ranges (between $16 \mathrm{GHz}$ and $20 \mathrm{GHz}$ ) have been exploited, thus the sub-carrier spacing can vary between $62.5 \mathrm{MHz}$ and $78.125 \mathrm{MHz}$; a cyclic prefix (CP) of about $2.1 \%$ of the symbol length is also added. The VCSEL bias current and the modulation amplitude in the following analysis have been optimized for single channel performance and set to $8 \mathrm{~mA}$ and $8.5 \mathrm{~mA}$ respectively. The SSB modulation is obtained by properly detuning a 25-GHz spacing WSS filter, which shows a 21-GHz full width half maximum (FWHM) [12]; the frequency detuning, around $9.5 \mathrm{GHz}$, allows to select half of the DMT dual sideband (DSB) spectrum while preserving the optical carrier, as shown in Figure $1 \mathrm{~b}$ ).

a)

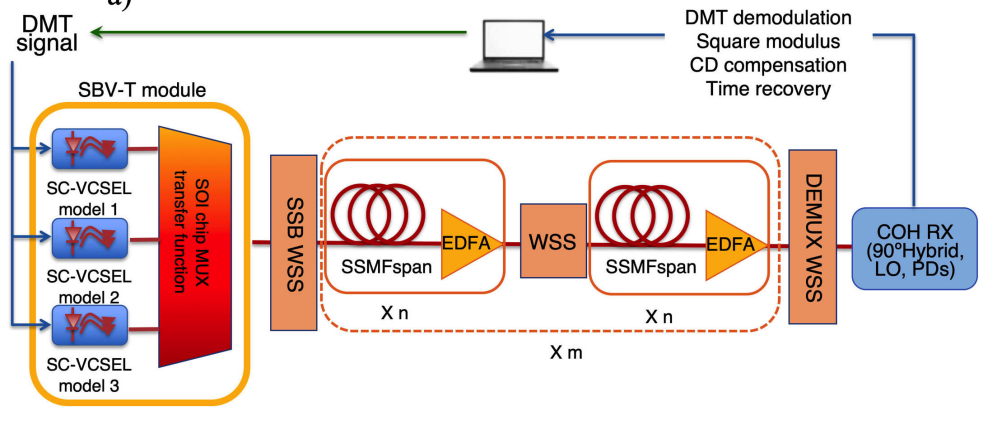

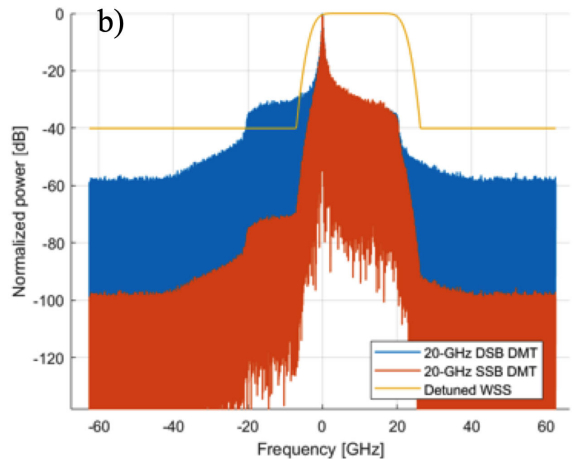

Figure 1. a) System model implemented in simulations. b) DMT spectra: DSB signal with 20-GHz electrical bandwidth (blue), SSB (red) obtained by a 9.5-GHz detuned WSS (orange) filtering.

In order to target transmission distances typical of MANs, we employ digital coherent detection (COHD) which allows easy CD DSP compensation, avoiding also the VCSEL chirp interplay with CD. The chirp associated with VCSEL DM will impair transmission performance only due to the presence of filtering associated to wavelength multiplexers and demultiplexers. Moreover, it has to be noticed that due to DM, the transmitted signal is just intensity modulated and then a simplified coherent receiver can be used [8]: after I and Q components recovery and CD compensation the I and Q square moduli are performed and summed up in order to obtain the originally transmitted intensity signal. This approach has the drawback to cancel the COHD advantages in terms of bit error rate (BER) as a function of signal to noise ratio (SNR) with respect to direct detection (DD), but avoids the use of phase and frequency recovery, reducing the complexity of the receiver DSP and also relaxing the constraints on VCSEL and local oscillator (LO) linewidths.

\subsection{Simplified COHD: impact on transmitted capacity of LO linewidth and drift}

Table I SSB DMT capacity in back to back as a function of LO linewidth, LO detuning with respect to the signal center frequency and OSNR.

\begin{tabular}{|c|c|c|c|c|}
\hline & LO linewidth & $-5 \mathrm{GHz}$ & $0 \mathrm{GHz}$ & $+5 \mathrm{GHz}$ \\
\hline \multirow{3}{*}{$\begin{array}{l}n \\
q \\
o \\
+ \\
\text { o } \\
z \\
0 \\
0\end{array}$} & $100 \mathrm{kHz}$ & $77.9 \mathrm{~Gb} / \mathrm{s}$ & $77 \mathrm{~Gb} / \mathrm{s}$ & $76.2 \mathrm{~Gb} / \mathrm{s}$ \\
\hline & $500 \mathrm{kHz}$ & $77.3 \mathrm{~Gb} / \mathrm{s}$ & $76.6 \mathrm{~Gb} / \mathrm{s}$ & $75.8 \mathrm{~Gb} / \mathrm{s}$ \\
\hline & $1 \mathrm{MHz}$ & $76.7 \mathrm{~Gb} / \mathrm{s}$ & $76.2 \mathrm{~Gb} / \mathrm{s}$ & $75.5 \mathrm{~Gb} / \mathrm{s}$ \\
\hline \multirow{3}{*}{ 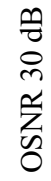 } & $100 \mathrm{kHz}$ & $58.7 \mathrm{~Gb} / \mathrm{s}$ & $58.1 \mathrm{~Gb} / \mathrm{s}$ & $57.4 \mathrm{~Gb} / \mathrm{s}$ \\
\hline & $500 \mathrm{kHz}$ & $58.4 \mathrm{~Gb} / \mathrm{s}$ & $57.7 \mathrm{~Gb} / \mathrm{s}$ & $57.3 \mathrm{~Gb} / \mathrm{s}$ \\
\hline & $1 \mathrm{MHz}$ & $58.1 \mathrm{~Gb} / \mathrm{s}$ & $57.3 \mathrm{~Gb} / \mathrm{s}$ & $57 \mathrm{~Gb} / \mathrm{s}$ \\
\hline
\end{tabular}

Table I in fact presents the results of preliminary simulations, in back to back condition, obtained when varying both LO linewidth and frequency mismatch with respect to the signal (LO shift), when the considered DM-VCSEL linewidth is $5 \mathrm{MHz}$. The LO power is $10 \mathrm{dBm}$ per state of polarization (SOP), and the photodiodes (PD) have 
$30 \mathrm{GHz}$ E/O bandwidth and a noise equivalent current (NEC) of $20 \mathrm{pA} / \mathrm{Hz}^{1 / 2}$. The results obtained for $30 \mathrm{~dB}$ and $40 \mathrm{~dB}$ optical SNRs (OSNRs) confirm that the impact of the LO linewidth is limited up to $1 \mathrm{MHz}$ and also the detuning of the LO with respect to the SSB spectrum center causes a modest impairment (in the $0 \mathrm{GHz}$ condition, a perfect superposition of LO and signal carrier is achieved). Actually, when the LO moves towards the signal higher frequencies (to the right in Figure1) the capacity slightly improves, whereas in the opposite direction it reduces as the beating signal starts to be filtered out by the PDs.

\section{IMPACT OF NODE FILTERING AND ADJACENT CHANNEL CROSSTALK}

In [9] we have evaluated the impact of node filtering on transmission capacities of DM-VCSELs employing DMT modulation considering single channel and single polarization transmission. In the following, we focus on SSB DMT transmission, which provides a higher spectral efficiency, and we take into account the presence of two adjacent channels to the channel under test, the VCSELs emitted respectively at $1540 \mathrm{~nm}$ (channel under test) $1539.8 \mathrm{~nm}$ and $1540.2 \mathrm{~nm}$ (see Figure $1 \mathrm{a}$ ). The propagation medium is a SSMF, with $0.25 \mathrm{~dB} / \mathrm{km}$ attenuation and $17 \mathrm{ps} / \mathrm{nm} \mathrm{km}$ dispersion at $1550 \mathrm{~nm}$, Erbium-doped fiber amplifiers (EDFAs) with 6-dB noise figure (NF) are used to compensate for the transmission losses. The filter transfer function employed in the simulations is the one measured at the output of a programmable optical filter (Finisar WaveShaper) emulating the WSS theoretical transfer function of [12]. It is worth recalling that at least two WSSes are needed for the analysis: one at the transmitter side (to implement the SSB modulation) and one at the receiver side to demodulate the channel under test.
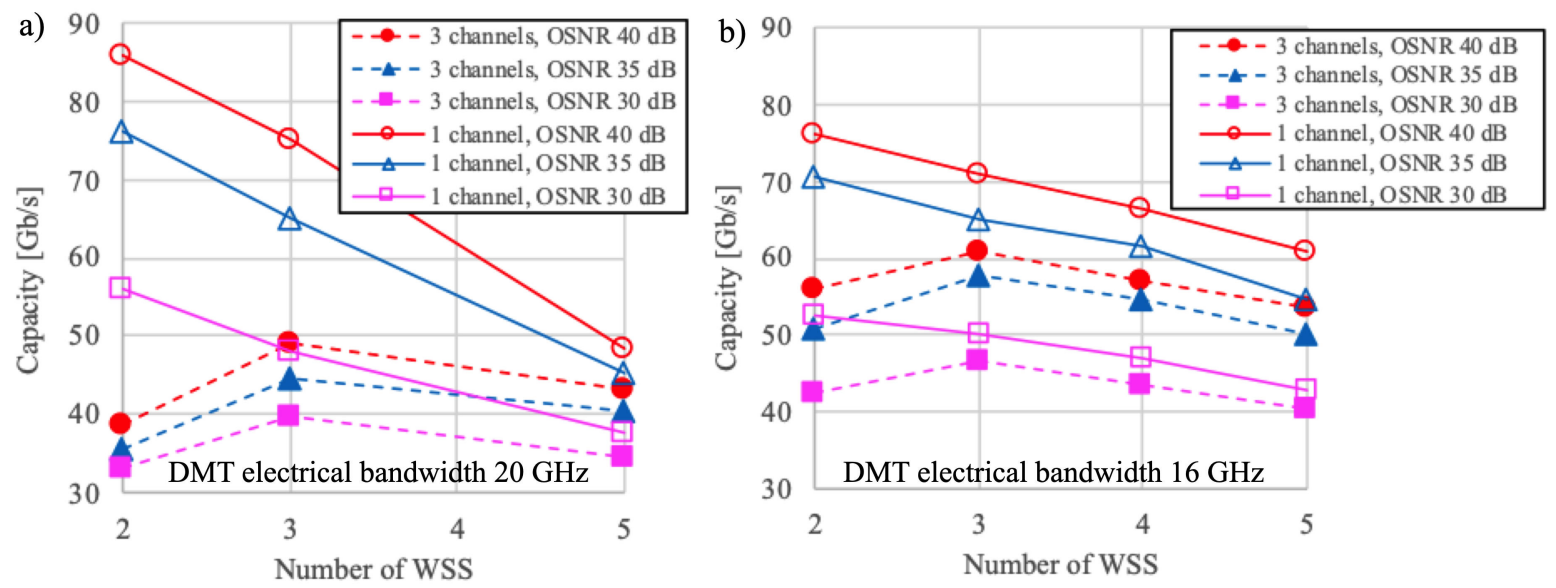

Figure 2 SSB DMT transmission capacities in 3-channel propagation vs number of crossed WSS for: 40-dB OSNR (red circle), 35-dB OSNR (blue triangle), 30-dB OSNR (pink square), 1 channel (continuous line, open symbol), 3 channels (dashed line, full symbol). a) Signal electrical bandit of $20 \mathrm{GHz}$. b) Signal electrical bandit of $16 \mathrm{GHz}$.
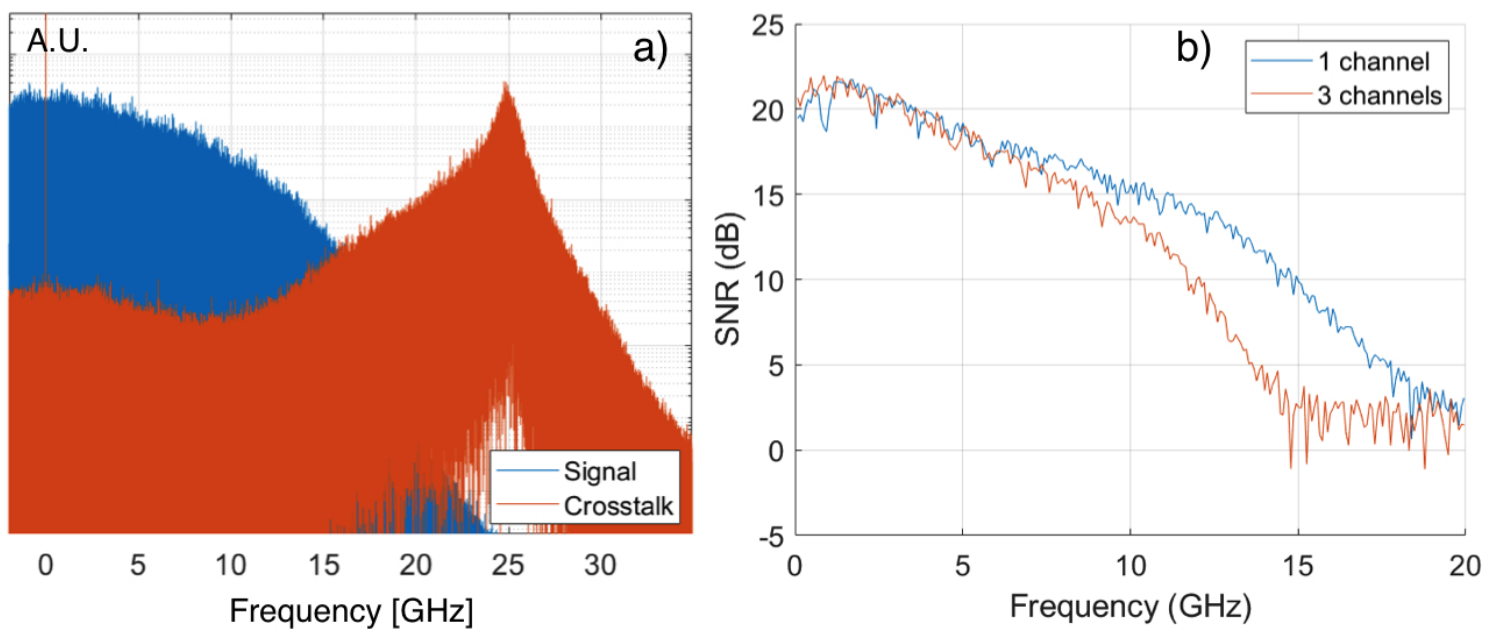

Figure 3 SSB DMT in 3-channel propagation: a) signal spectrum after 2 WSS (blue) and crosstalk contribution (red) b) corresponding per subcarrier received SNR.

Figure 2 (a), shows the transmitted capacity as a function of the number of crossed WSSes for three OSNR values, when the electrical bandwidth filled up by the DMT signal is equal to the VCSEL bandwidth, i.e. $20 \mathrm{GHz}$. 
As can be seen by comparing continuous lines (single channel) and dashed curves ( 3 channels) the presence of the linear crosstalk reduces the capacity of the 2 WSS case of nearly $50 \%$, whereas for a higher number of filters the reduction is less consistent. This is caused by the presence of a residual component of the adjacent channel located on the higher frequency subcarriers (see Figure $3 \mathrm{a}$ )), which lowers those subcarriers SNR, that is not sufficient to support high-level modulation formats (Figure $3 \mathrm{~b}$ ). We have then evaluated to reduce the DMT electrical signal bandwidth and the best results have been obtained with $16 \mathrm{GHz}$, a further bandwidth decrement being detrimental. Figure $2 \mathrm{~b}$ ) in fact shows that the improvement on the capacity performance induced by the crosstalk reduction is a higher than the impairment due to the bandwidth limitation: the single channel capacity is reduced by $10 \%$ but the 3-channel capacity improves of nearly $20 \%$.

\section{CONCLUSIONS}

We analyzed the performance of multi-Tb/s transmitter modules with $25-\mathrm{GHz}$ WDM granularity obtained aggregating multiple C-band DM-VCSELs in SOI chips. Adopting direct modulation with advanced modulation formats, such as DMT, more than $50 \mathrm{~Gb} / \mathrm{s}$ per SOP per channel can be achieved.

The combination with simplified coherent detection allows to bridge MAN typical link distances with very low capacity reductions even with $\mathrm{LO}$ with $1-\mathrm{MHz}$ linewidth with respect to $100-\mathrm{kHz}$ one.

We demonstrated that a reduction of the DMT electrical spectrum occupancy can be beneficial in case of crosstalk induced by adjacent channels with tight WDM spacing, despite single channel lower performance. Limiting the DMT bandwidth to $16 \mathrm{GHz}$, also in presence of numerous hops between nodes inside the MAN, the transported capacity is higher than $50 \mathrm{~Gb} / \mathrm{s}$ per SOP per channel for OSNR higher than $35 \mathrm{~dB}$; these OSNR values can guarantee more than $200 \mathrm{~km}$ reach with $35-\mathrm{km}$ spans in the envisaged MAN [9].

Indeed, as the crosstalk is mainly due to the spectrum broadening induced by the chirp associated with DM, to further improve the performance in future work we will investigate the reduction of the modulation amplitude and also the use of some filtering at DSP level.

\section{ACKNOWLEDGEMENTS}

This work has been supported by the H2020 EU PASSION Project (GA 780326).

\section{REFERENCES}

[1] M. S. Moreolo et al., " Spectrum/Space Switching and Multi-Terabit Transmission in Agile Optical Metro Networks," in OECC/PSC 2019 Proceedings, C000235.

[2] M. S. Moreolo et al., "Modular SDN-enabled S-BVT Adopting Widely Tunable MEMS VCSEL for Flexible/Elastic Optical Metro Networks," in Proceedings OFC 2018, San Diego, CA, paper M1A.7, March 2018.

[3] C. Xie, et al., "Single VCSEL 100-Gb/s short reach system using discrete multi-tone modulation and direct detection," in Proceedings OFC 2015, Los Angeles, CA, paper Tu2H.2, March 2015.

[4] A. Gatto, et al., "Beyond $25 \mathrm{~Gb} / \mathrm{s}$ Directly-Modulated Widely Tunable VCSEL for Next Generation Access Network," in Proceedings OFC 2018, San Diego, CA, paper Th1E.2, March 2018.

[5] A. Gatto, D. Argenio, P. Boffi "Very high-capacity short-reach VCSEL systems exploiting multicarrier internsity modulation and direct detection" Opt. Express, 24, 12, 12769-12775 (2016)

[6] S. Bhat, M. Harjanne, F. Sun, et al. "Low Loss Devices fabricated on the Open Access 3mm SOI Waveguide Platform at VTT" in European Conference on Integrated Optics 2019, Ghent, Belgium, 2019, paper T.Po2.18

[7] A. Boletti, P. Boffi, P. Martelli, M. Ferrario, M. Martinelli "Performance analysis of communication links based on VCSEL and silicon photonics technology for high-capacity data-intensive scenario" Opt. Express, 23, 1806-1815 (2015)

[8] C. Xie, S. Spiga, P. Dong, et al., "400-Gb/s PDM-4PAM WDM System Using a Monolithic 2×4 VCSEL Array and Coherent Detection," J. Lightwave Technol., 33, (3), pp. 670-677, 2015.

[9] M. Rapisarda, et al. "Impact of Chirp in High-Capacity Optical Metro Networks Employing DirectlyModulated VCSELs," Photonics, 5, (4), pp. 51, 2018.

[10] P. S. Chow et al., "A practical discrete multitone transceiver loading algorithm for data transmission over spectrally shaped channels," IEEE Trans. on Comm., 43, 773-775, 1995.

[11] M. Svaluto Moreolo, et al. "SDN-enabled sliceable BVT based on multicarrier technology for multi-flow rate/distance and grid adaptation.” J. Lightwave Technol., 34, 1516-1522, 2016.

[12] C. Pulikkaseril, L.A. Stewart, M.A.F. Roelens, G.W. Baxter, S. Poole, S. Frisken, "Spectral modeling of channel band shapes in wavelength selective switches." Opt. Express, 19, 8458-8470, 2011. 